\title{
Preferential Influx and Decreased Fractional Loss of Lipoprotein(a) in Atherosclerotic Compared with Nonlesioned Rabbit Aorta
}

\author{
Lars B. Nielsen, ${ }^{\star}$ Steen Stender, ${ }^{\ddagger}$ Matti Jauhiainen, ${ }^{\S}$ and Børge G. Nordestgaard

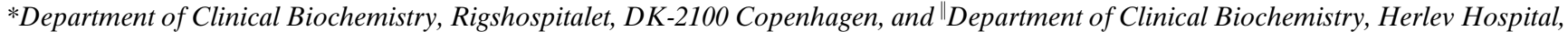 \\ DK-2730 Herlev, University of Copenhagen, Denmark; ${ }^{\circledR}$ National Public Health Institute, Department of Biochemistry, FIN-00300 \\ Helsinki, Finland; and ${ }^{\ddagger}$ Clinical Institute, University of Odense, DK-5000 Odense, Denmark
}

\begin{abstract}
The aim was to investigate the atherogenic potential of lipoprotein(a) $(\mathrm{Lp}(\mathrm{a}))$ and to further our understanding of the atherogenic process by measuring rates of transfer into the intima-inner media (i.e., intimal clearance) and rates of loss from the intima-inner media (i.e., fractional loss) of $L p(a)$ and LDL using cholesterol-fed rabbits with nonlesioned $(n=13)$ or atherosclerotic aortas $(n=12)$. In each rabbit, ${ }^{131} \mathrm{I}-\mathrm{Lp}$ (a) (or ${ }^{131} \mathrm{I}-\mathrm{LDL}$ ) was injected intravenously $26 \mathrm{~h}$ before and ${ }^{125} \mathrm{I}-\mathrm{Lp}(\mathrm{a})$ (or $\left.{ }^{125} \mathrm{I}-\mathrm{LDL}\right) 3 \mathrm{~h}$ before the aorta was removed and divided into six consecutive segments of similar size. The intimal clearance of $\mathrm{Lp}(\mathrm{a})$ and LDL was similar and markedly increased in atherosclerotic compared with nonlesioned aortas (ANOVA, effect of atherosclerosis: $P<$ 0.0001). Fractional losses of labeled Lp(a) and labeled LDL in atherosclerotic aorta were on average 25 and $43 \%$, respectively, of that in nonlesioned aortas (ANOVA, effect of atherosclerosis: $P<0.0001)$. Fractional loss of $\mathrm{Lp}(\mathrm{a})$ was $73 \%$ of that of LDL (ANOVA, effect of type of lipoprotein: $P=0.07)$. These data suggest that the development of atherosclerosis is associated with increased influx as well as decreased fractional loss of $\mathrm{Lp}(\mathrm{a})$ and $\mathrm{LDL}$ from the intima. Accordingly, Lp(a) may share with LDL the potential for causing atherosclerosis. (J. Clin. Invest. 1996. 98:563-571.) Key words: atherosclerosis • cholesterol • efflux • fractional loss $\bullet$ low-density lipoprotein
\end{abstract}

\section{Introduction}

High plasma levels of LDL are causally related to development of atherosclerosis (1); LDL carries the major fraction of apo B and cholesterol in plasma. The importance for development of atherosclerosis of the lipoprotein(a) $(\mathrm{Lp}(\mathrm{a}))^{1}$ particle, which is another apo $\mathrm{B}$ and cholesterol-containing lipoprotein fraction, is less well understood $(1,2)$. However, in most casecontrol studies, high plasma levels of Lp(a) were associated

Address correspondence to Børge G. Nordestgaard, Department of Clinical Biochemistry, Herlev Hospital, DK-2730 Herlev, Denmark. Phone: +45 445353 00, ext. 3843; FAX: + 454453 53 32; or Lars Bo Nielsen, Gladstone Institute of Cardiovascular Disease, P.O. Box 419100, San Francisco, CA 94141-9100. Phone: 415-826-7500; FAX: 415-285-5632.

Received for publication 30 September 1995 and accepted in revised form 3 May 1996.

1. Abbreviation used in this paper: $\mathrm{Lp}(\mathrm{a})$, Lipoprotein(a).

J. Clin. Invest.

(C) The American Society for Clinical Investigation, Inc.

0021-9738/96/07/0563/09 \$2.00

Volume 98, Number 2, July 1996, 563-571 with an increased risk of atherosclerosis-related disease, and in five of seven prospective studies, plasma Lp(a) levels were higher in atherosclerosis-effected individuals than in controls (for review see reference 2). The transgenic mice expressing human apo(a) also developed more severe atherosclerosis than control mice (3).

LDL- and Lp(a)-like particles have predominantly been detected in the vessel wall at sites of atherosclerosis $(4,5)$. The concentration of the $\mathrm{Lp}(\mathrm{a})$ apo(a) in vein grafts was $240 \%$ that of apo B, when compared with the concentrations of the two apolipoproteins in plasma (6). More Lp(a) than LDL was extractable after plasmin digestion of human arterial intima (7), and autoradiographic studies in mice suggest that $\mathrm{Lp}(\mathrm{a})$ accumulates in the arterial intima preferentially to LDL (8). Whether these observations reflect increased influx or decreased efflux in the vessel wall remains to be determined.

The bulk of evidence supports the idea that accumulation of plasma-derived atherogenic lipoproteins in the intima constitutes a fundamental event in the initiation of atherosclerotic lesions (9). It is therefore important to further our understanding of the interaction of plasma lipoproteins with the arterial intima in vivo. A specific aim of the present study was to compare the atherogenic potential of $\mathrm{Lp}(\mathrm{a})$ and LDL: rates of transfer into intima-inner media (i.e., intimal clearance) and rates of loss from intima-inner media (i.e., fractional loss) of labeled $\mathrm{Lp}(\mathrm{a})$ and LDL were determined in vivo in nonlesioned and atherosclerotic aortas of cholesterol-fed rabbits.

\section{Methods}

Animals. Male white rabbits (Danish Country Strain, Statens Seruminstitut, Copenhagen, Denmark) weighing 2.8 to $4.0 \mathrm{~kg}$ received $100 \mathrm{~g}$ of chow per day containing 94 to $94.75 \%$ standard rabbit pellets (Altromin 2113; Lage, Germany), 5\% corn oil (Oleum Maides; Mecobenzon, Copenhagen, Denmark) and 0.25 to $1 \%$ cholesterol (USP-cholesterol; Sigma Chemical Co., Copenhagen, Denmark).

To induce atherosclerosis in the aorta, 12 rabbits ( 6 used for LDL studies and 6 for $L p(a)$ studies) were fed this chow for 173-236 d; the amount of cholesterol in the chow was adjusted regularly to maintain a plasma cholesterol level of 30-70 $\mathrm{mmol} / \mathrm{liter}$. These 12 rabbits all had visible atherosclerotic lesions in the aorta. The severity of atherosclerosis in aortic segments was quantified by measuring the cholesterol content of the intima-inner media; the aortic cholesterol content is closely associated with other indices of atherosclerosis, in rabbits and pigs $(10,11)$.

To increase the plasma LDL-cholesterol level without inducing atherosclerosis, 13 other rabbits ( 7 used to study LDL and 6 to study $\mathrm{Lp}(\mathrm{a}))$ were fed the $1 \mathrm{~g}$ cholesterol/d chow for 6-10 $\mathrm{d}$. None of these rabbits had visible lesions in the aorta.

The experimental protocol was approved by the Danish government body supervising animal experiments (the Animal Experiment Inspectorate).

Isolation of human $L p(a)$ and $L D L$. As described previously (12), $\mathrm{Lp}(\mathrm{a})$ concentrations in human plasma, isolated $\mathrm{Lp}(\mathrm{a})$ preparations, and rabbit plasma were determined using a turbidimetric assay or 
rocket immunoelectrophoresis. LDL protein concentration was estimated from the absorbance at $220 \mathrm{~nm}$ before iodination (12).

For the isolation of $\mathrm{Lp}(\mathrm{a})$, plasma $\mathrm{Lp}$ (a) concentration was determined in at least 10 healthy subjects. $\mathrm{Lp}(\mathrm{a})$ was subsequently isolated from pooled plasmas from the two subjects with the highest $\mathrm{Lp}(\mathrm{a})$ concentrations $(0.38-0.58 \mathrm{mg} / \mathrm{ml})$, as described in detail elsewhere (13). The apo(a) isoforms of the donors were S2/S5 and S3 for one of the two preparations of $\mathrm{Lp}(\mathrm{a})$ and S1/S5 and S2 for the other; apo(a) isoforms were determined essentially as described by Utermann et al. (14).

LDL $(1.019<d<1.063 \mathrm{~g} / \mathrm{ml})$ was isolated from the plasma of a donor with low plasma $\mathrm{Lp}(\mathrm{a})$ by the use of sequential ultracentrifugation (15). The contamination of purified LDL with $\mathrm{Lp}(\mathrm{a})$ was less than $0.2 \%$ of the total lipoprotein mass; total $\mathrm{Lp}(\mathrm{a})$ mass was compared with total LDL mass.

Lipoprotein labeling. Two separate aliquots of purified $\mathrm{Lp}(\mathrm{a})(2$ $\mathrm{ml}, 6.2-9.6 \mathrm{mg}$ total $\mathrm{Lp}(\mathrm{a}))$ or purified LDL (0.6-1.2 $\mathrm{ml}, 5 \mathrm{mg}$ LDL protein $\approx 22 \mathrm{mg}$ total LDL, since protein constitutes $22.4 \%$ of total LDL mass, reference 16) were labeled with $75-260 \mathrm{MBq}{ }^{125} \mathrm{I}$ and 90 $260 \mathrm{MBq}{ }^{131} \mathrm{I}$, respectively, using the iodine monochloride method $(17,18)$ as described previously $(12,15)$. To remove unbound iodine, labeled lipoprotein preparations were passed through a PD-10 column (Pharmacia LKB Biotechnology Inc., Piscataway, NJ) before 100-160 mg of rabbit albumin (Sigma Chemical Co., St. Louis, MO) was added. The iodination efficiency averaged $57 \%$ and was similar for the two lipoproteins. The specific activities were 0.2 to $1.2 \times 10^{9}$ cpm ${ }^{125} \mathrm{I}$ per mg $\mathrm{Lp}(\mathrm{a}), 0.1$ to $1.0 \times 10^{9} \mathrm{cpm}{ }^{131} \mathrm{I}$ per $\mathrm{mg} \mathrm{Lp}(\mathrm{a}), 0.2$ to $2.5 \times 10^{9} \mathrm{cpm}{ }^{125} \mathrm{I}$ per $\mathrm{mg}$ LDL, and 0.1 to $0.8 \times 10^{9} \mathrm{cpm}{ }^{131} \mathrm{I}$ per $\mathrm{mg}$ LDL. 96 to $98 \%$ of the radioactivity in both labeled Lp(a) and LDL was TCA precipitate. Less than $3.3 \%$ of the TCA-precipitable radioactivity was extractable with chloroform-methanol (1:1, vol/vol). Labeled lipoprotein preparations were passed through $0.22-$ or $0.45-\mu \mathrm{m}$ filters and used for injection within $48 \mathrm{~h}$ of labeling.

Lipoprotein injection. In six rabbits with nonlesioned aortas and six rabbits with atherosclerotic aortas, ${ }^{131} \mathrm{I}-\mathrm{Lp}(\mathrm{a})\left(3.4 \pm 0.9 \times 10^{8} \mathrm{cpm}\right.$ per kg body wt) was injected intravenously $26.4 \pm 0.2 \mathrm{~h}$ before and ${ }^{125} \mathrm{I}$-Lp(a) $\left(2.7 \pm 0.7 \times 10^{8} \mathrm{cpm}\right.$ per kg body wt) $3.0 \pm 0.04 \mathrm{~h}$ before the aorta was removed. Similarly, in seven rabbits with nonlesioned aortas and in six rabbits with atherosclerotic aortas, ${ }^{131} \mathrm{I}-\mathrm{LDL}(2.0 \pm 0.4 \times$ $10^{9} \mathrm{cpm}$ per kg body wt) was injected $26.1 \pm 0.3 \mathrm{~h}$ before and ${ }^{125} \mathrm{I}$-LDL $\left(7.0 \pm 2.0 \times 10^{8} \mathrm{cpm}\right.$ per kg body wt) $3.0 \pm 0.02 \mathrm{~h}$ before the aorta was removed.

The rabbits used to study $\mathrm{Lp}$ (a) kinetics received an intravenous injection of 7-10 $\mathrm{ml}$ human $d<1.12 \mathrm{~g} / \mathrm{ml}$ lipoproteins (containing 29$38 \mathrm{mg} \mathrm{Lp}(\mathrm{a}))$ immediately before injection of ${ }^{131} \mathrm{I}-\mathrm{Lp}(\mathrm{a})$. The $d<1.12$ $\mathrm{g} / \mathrm{ml}$ lipoprotein fraction was prepared using the same batch of human $\mathrm{Lp}(\mathrm{a})$ - rich plasma as was used for isolation of $\mathrm{Lp}(\mathrm{a})$ for labeling (12).

Blood samples of $1 \mathrm{ml}$ were drawn from an ear vein at regular intervals after injection of labeled lipoproteins.

Removal of aorta. Each rabbit was injected intravenously with pentobarbital (50-100 mg/kg) before the thoracic cavity was opened and a cannula was placed in the left ventricle of the heart, through which the vascular bed was perfused with 1 liter cold saline. The aorta was removed and freed of adventitial tissue. The lumen was flushed with cold saline several times during this procedure to chill the aortic tissue. The vessel was opened longitudinally, rinsed further with cold saline, and divided into the aortic arch (from the heart to the first intercostal branches), the thoracic aorta (to the celiac axis), and the abdominal aorta (to the iliac bifurcation). Each of these three parts were then divided into a proximal and a distal segment of similar size. The intima-inner media in each of the six aortic segments was stripped from the outer media and minced at $4{ }^{\circ} \mathrm{C}$.

Trichloroacetic acid precipitable radioactivity. Minced tissues and aliquots of plasma and diluted doses were TCA precipitated after the addition of albumin as described (15). The time between removal of aorta and precipitation of proteins in the intima-inner media was 30 $45 \mathrm{~min}$. Radioactivity was determined in a double channel gamma counter (LKB compugamma 1282; Wallac, Copenhagen, Denmark). Tissues were counted for at least $42 \mathrm{~min}$; typical standard errors of counting rates of TCA-precipitable ${ }^{125} \mathrm{I}$ and ${ }^{131} \mathrm{I}$ in aortic intima-inner media were $<2 \%$.

Analysis. Lipids in TCA-precipitates of aortic intima-inner media were extracted over a 24-h period with chloroform/methanol (2:1, $\mathrm{vol} / \mathrm{vol}$; $\sim 1 \mathrm{ml}$ solvent per $50 \mathrm{mg}$ tissue) followed by two further extractions with chloroform/methanol $(1: 1, \mathrm{vol} / \mathrm{vol})$ before the combined lipid extract was washed by the Folch procedure (19). Cholesterol was quantified with the Liebermann-Burchard method (20) or with an enzymatic method, which measures total cholesterol (CHODPAP; Boehringer Mannheim, Mannheim, Germany) after evaporation of the chloroform/methanol and solubilization of the extract in isopropanol. When cholesterol in lipid extracts of 66 TCA-precipitated aortic segments was determined by both methods, similar results were obtained: on linear regression analysis, Pearson's correlation coefficient $(r)$ for the association between values obtained by the two methods was $>0.98$ and the slope and $y$-intercept of the regression line were not significantly different from 1 and 0 , respectively. The CHOD-PAP enzymatic method was also used for determination of cholesterol in plasma and lipoprotein fractions.

Agarose gel electrophoresis ( $0.5 \%$ agarose), nondenaturating polyacrylamide gradient gel electrophoresis, and two-tier rocket immunoelectrophoresis were performed as previously described (12, $21)$. The recovery of radioactivity on the two-tier rocket immunoelectrophoresis gel was $92.2 \pm 1.2 \%(n=78 ; 8$ aliquots of doses and 70 plasma samples).

Calculations of intimal clearance and fractional loss. TCA-precipitable radioactivity in tissues, washes, plasma, and doses were used in the calculations. Intimal clearance and fractional loss of labeled $\mathrm{Lp}(\mathrm{a})$ and LDL were calculated under the assumption that the kinetics of newly entered lipoproteins in the intima-inner media can be described by a one-pool compartment model; i.e., the loss of labeled lipoproteins from the intima-inner media increases linearly with the amount of labeled lipoproteins in the compartment. This assumption has previously been tested and was found to be plausible for iodine labeled LDL in nonlesioned and atherosclerotic aortas of rabbits (22) and monkeys (23). In the one-pool compartment model, the kinetics of labeled lipoproteins can be described by the equation (23-25):

$\frac{d A}{d t}=k_{i} c(t)-k_{e} A(t)$

with two unknowns, intimal clearance $\left(k_{\mathrm{i}}\right.$, in $\mathrm{nl} / \mathrm{cm}^{2}$ per h) and fractional loss $\left(k_{e}\right.$, in $\left.\mathrm{h}^{-1}\right) . A(t)$ is the aortic radioactivity $\left(\mathrm{cpm} / \mathrm{cm}^{2}\right)$ at time $t$ and $c(t)$ is the concentration of radioactivity in plasma $(\mathrm{cpm} / \mathrm{nl})$ at time $t$. Plasma radioactivity curves were fitted to biexponential functions:

$c(t)=C_{1} e^{-b_{1} t}+C_{2} e^{-b_{2} t}$

where $C_{1}$ and $b_{1}$ are constant and slope for the initial rapid removal of radioactivity from plasma and $C_{2}$ and $b_{2}$ are constant and slope for the subsequent slow removal of radioactivity from plasma. After substitution of Eq. 2 into Eq. 1, Eq. 1 can be solved to obtain:

$A(t)=k_{\mathrm{i}}\left(C_{1} \frac{\left(e^{-k_{\mathrm{e}} t}-e^{-b_{1} t}\right)}{\left(b_{1}-k_{\mathrm{e}}\right)}+C_{2} \frac{\left(e^{-k_{\mathrm{e}} t}-e^{-b_{2} t}\right)}{\left(b_{2}-k_{\mathrm{e}}\right)}\right)$

Since the rabbits were given two differently labeled aliquots of the same lipoprotein but at different time intervals before removal of the aorta, Eq. 3 can be written for each isotope: fractional loss $\left(k_{\mathrm{e}}\right)$ and intimal clearance $\left(k_{\mathrm{i}}\right)$ can then be calculated by solving two equations with two unknowns.

Plasma and arterial wall radioactivity values are subject to measurement error. Two previous studies have estimated uncertainties in calculated values of intimal clearance and fractional loss that are induced by measurement uncertainties of the order likely in practice $(24,26)$, using experimental conditions quite similar to the present study. Wootton et al. (24) appplied random noise with zero mean and $1.5 \% \mathrm{SD}$ to plasma radioacitvity data and random noise of zero mean and $5 \%$ SD to arterial wall radioactivity data, which produced uncertainties of $7 \%$ SD in calculated intimal clearance and $16 \%$ SD in cal- 
culated fractional loss. Schwenke and Zilversmit (26) estimated that a $5 \%$ error in arterial wall radioactivity would produce an 8.5 to $14.5 \%$ error in calculated fractional loss and a 1.5 to $10 \%$ error in calculated intimal clearance (data calculated from Table 2 in reference 26).

In the $\mathrm{Lp}(\mathrm{a})$ experiments, some of the radioactivity in plasma was in $\mathrm{Lp}$ (a) particles that had lost the apo(a) moiety; i.e., Lp(a)-. Accordingly, the combined transfer of $L p(a)$ and $L p(a)-$ between plasma and the arterial wall was studied when total (TCA-precipitable) radioactivity was used to solve Eq. 3 . Since $\mathrm{Lp}(\mathrm{a})$ - shares properties with LDL $(27,28)$, the following calculations were performed to correct values of $\mathrm{Lp}(\mathrm{a})$ fractional loss and intimal clearance for the presence of $\mathrm{Lp}(\mathrm{a})-$. The relative amount of radioactivity in $\mathrm{Lp}(\mathrm{a})-$ $10 \mathrm{~min}, 3 \mathrm{~h}$ and $26 \mathrm{~h}$ after intravenous injection of $\mathrm{Lp}(\mathrm{a})$ was determined in each rabbit using two-tier rocket immunoelectrophoresis. The relative amount of radioactivity in $\mathrm{Lp}(\mathrm{a})$ - at other time points was estimated by linear interpolation. The plasma $L p(a)-$ radioactivity curve was then fitted to third-degree polynomials and the contribution of $\mathrm{Lp}(\mathrm{a})$ - to the arterial wall radioactivity at both 3 and $26 \mathrm{~h}$ was calculated in each rabbit as previously described (25). In these calculations, it was assumed that the intimal clearance of $\operatorname{Lp}(\mathrm{a})-$ is similar to that of LDL (and Lp(a)), and that fractional loss of Lp(a)is similar to that of LDL: the Lp(a) - intimal clearance was that determined for $\mathrm{Lp}(\mathrm{a})$ in the same rabbit on the basis of total amounts of TCA-precipitable radioactivity in plasma and aortic intima-inner media. Fractional loss of $\mathrm{Lp}(\mathrm{a})$ - in a given aortic segment of nonlesioned and atherosclerotic aortas was assumed to be the average fractional loss of LDL in that same segment of nonlesioned or atherosclerotic aortas in the LDL experiments. After subtraction of $\mathrm{Lp}(\mathrm{a})$ - radioactivity from total $\mathrm{Lp}(\mathrm{a})$ radioactivity in plasma and arterial tissue, corrected intimal clearance and corrected fractional loss for pure $\mathrm{Lp}(\mathrm{a})$ were calculated based on the arterial wall and plasma radioactivity that was attributable only to $L p(a)$. Since labeled free apo(a) was not detected in plasma of rabbits injected with labeled $\mathrm{Lp}(\mathrm{a})$, no corrections for the possible contribution of labeled free apo(a) to arterial wall radioactivity were, or could be, made.

Calculations of crude accumulation and crude fractional loss. Crude accumulation in aorta after 3- and 26-h exposures (in $\mathrm{nl} / \mathrm{mg}$ wet $\mathrm{wt}$ ) to the labeled lipoproteins was calculated as radioactivity in intima-inner media (in $\mathrm{cpm} / \mathrm{mg}$ wet wt), divided by the mean plasma radioactivity concentration during the experimental period (in $\mathrm{cpm} / \mathrm{nl}$ ).

Crude fractional loss was calculated as:

$$
1-\frac{\text { crude accumulation }_{\text {long }}}{\text { crude accumulation }_{\text {short }} \times\left(\frac{t_{\text {long }}}{t_{\text {short }}}\right)}
$$

in which $t$ is the exposure period (in h). Suffixes "long" and "short" refer to 26- and 3-h exposure periods, respectively. The calculations of crude accumulation and crude fractional loss do not depend on the assumption of a one-pool compartment.

Statistics. Arterial wall parameters were evaluated using a twoway (aortic cholesterol in atherosclerotic aortas) or a three-way (all other parameters) layout ANOVA (29) with random effects (30). In this model, for a given variable, each rabbit has its own level: the random effects describe variations between rabbits. The model allows differences in variances between atherosclerotic and nonlesioned aortas and in the model for crude accumulation for $3 \mathrm{~h}$. The model furthermore was altered to allow differences in variances between different aortic sites. The contributions of different aortic sites (segments 1-6), the type of lipoprotein ( $\mathrm{Lp}(\mathrm{a})$ or LDL), and the presence or absence of atherosclerosis (nonlesioned or atherosclerotic) to the total variation were assessed using the "proc mixed" procedure in the statistical program (6.09 ed.; SAS Institute, Cary, NC). Aortic cholesterol, intimal clearance, fractional loss, crude accumulation during 3 and $26 \mathrm{~h}$, and crude fractional loss were all transformed logarithmically before statistical analysis, to approximate normal distributions. Initially, an ANOVA model was built to include all main effects (aortic site, type of lipoprotein, presence or absence of atherosclerosis) and all two-factor interactions (aortic site by type of lipoprotein, type of lipoprotein by atherosclerosis, aortic site by atherosclerosis). In the two-way layout, ANOVA aortic site, type of lipoprotein, and the aortic site by type of lipoprotein interaction were included. Using a stepdown procedure, the model was reduced as much as possible; reduction in $-2 \log$ likelihood determined if the main effects or interaction terms could be removed. If the reduction in $-2 \log$ likelihood was significant ( $x^{2}$ distribution with 1 degree of freedom), when a term was removed, that term remained in the model. The significance level upon removal from the model determined the effect of a term; main effects were only tested when removed from a model without interaction terms involving the main effect under investigation. Differences in variances were also tested for significance using reduction in -2 $\log$ likelihood. Student's $t$ test was used to test differences between means in two group comparisons. $P$ values are two tailed. Values are presented as means \pm SEM.

\section{Results}

Table I shows plasma lipoprotein concentrations and length of the cholesterol feeding period in rabbits used to study accumulation of labeled lipoproteins in the intima-inner media.

Labeled Lp(a) and LDL, visualized with autoradiography, comigrated with their respective nonlabeled lipoproteins, visualized with Coomassie blue staining, on nondenaturating 2.5$16 \%$ polyacrylamide gradient gels and $0.5 \%$ agarose gels (Fig.

Table I. Lipoprotein Concentrations and Duration of Cholesterol Feeding Period in the Rabbits with Nonlesioned or Atherosclerotic Aortas

\begin{tabular}{|c|c|c|c|c|c|c|c|}
\hline & \multirow{2}{*}{$\begin{array}{l}\text { Number of } \\
\text { rabbits }\end{array}$} & \multicolumn{4}{|c|}{ Plasma cholesterol } & \multirow[b]{2}{*}{$\mathrm{Lp}(\mathrm{a})^{*}$} & \multirow{2}{*}{$\begin{array}{c}\text { Period of } \\
\text { cholesterol feeding }\end{array}$} \\
\hline & & Total & VLDL + IDL & LDL & HDL & & \\
\hline & & \multicolumn{4}{|c|}{$\mathrm{mmol} /$ liter } & $m g / m l$ & $d$ \\
\hline \multicolumn{8}{|l|}{ LDL studies } \\
\hline Rabbits with nonlesioned aortas & 7 & $21.2 \pm 2.9$ & $15.8 \pm 2.2$ & $4.8 \pm 0.8$ & $0.6 \pm 0.1$ & $\mathrm{ND}^{\S}$ & $7 \pm 1$ \\
\hline Rabbits with atherosclerotic aortas & 6 & $58.3 \pm 9.4$ & $46.6 \pm 6.5$ & $11.0 \pm 3.3$ & $0.8 \pm 0.1$ & ND & $180 \pm 2$ \\
\hline \multicolumn{8}{|l|}{ Lp(a) studies } \\
\hline Rabbits with nonlesioned aortas & 6 & $17.1 \pm 3.4$ & $11.3 \pm 3.2$ & $5.2 \pm 0.4$ & $0.6 \pm 0.1$ & $0.27 \pm 0.03$ & $6 \pm 0$ \\
\hline Rabbits with atherosclerotic aortas & 6 & $31.2 \pm 3.1$ & $24.3 \pm 2.7$ & $6.3 \pm 0.6$ & $0.6 \pm 0.1$ & $0.23 \pm 0.05$ & $229 \pm 3$ \\
\hline
\end{tabular}

Values are means \pm SEM. *Initial Lp(a) plasma concentration after intravenous injection of $d<1.12 \mathrm{~g} / \mathrm{ml}$ lipoproteins. ${ }^{*}$ Plasma total and lipoprotein cholesterol values are at the end of the experiment. ${ }^{\S} \mathrm{ND}$, not determined: rabbits used for LDL studies were not injected with $d<1.12 \mathrm{~g} / \mathrm{ml}$ lipoproteins. 

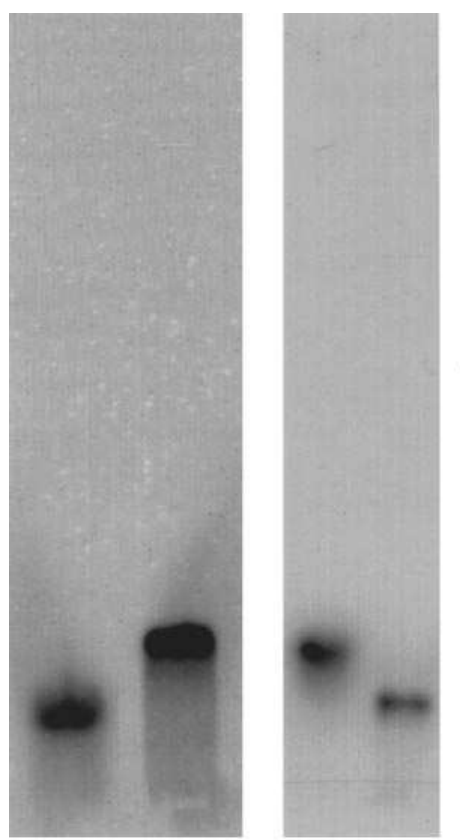

\section{Apo(a)}
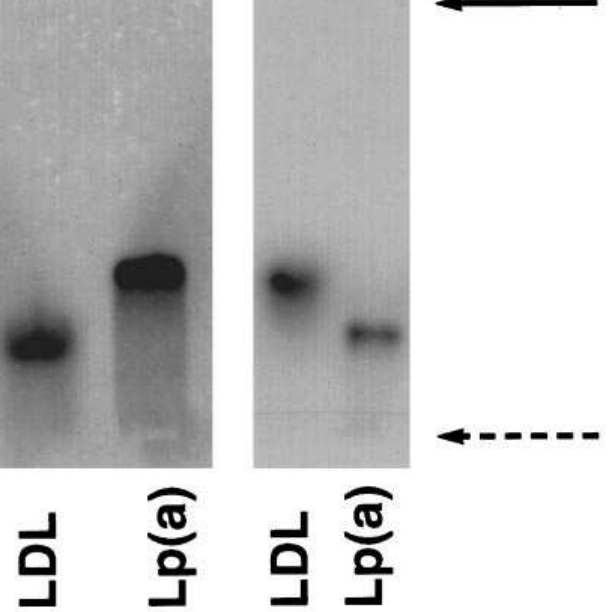

Figure 1. Autoradiographs of agarose electrophoresis gels $(0.5 \%)$ (left two lanes) and nondenaturating polyacrylamide gradient gels (2.5-16\%, Isolab Inc., Akron, OH) (right two lanes) of ${ }^{131} \mathrm{I}-\mathrm{Lp}(\mathrm{a})$ and ${ }^{125}$ I-LDL. Lipoproteins were loaded at the bottom (dotted arrow). The migration distance of free apo(a) (solid arrow) on nondenaturating polyacrylamide gradient gels was determined in another electrophoresis, but under similar conditions $(21,27)$.

1). Isolated $\mathrm{Lp}(\mathrm{a})$ and LDL migrated as two distinct bands and no labeled free apo(a) was detected. On gel filtration chromatography, no disintegration products of labeled $L p(a)$ were observed (Fig. 2).

$26 \mathrm{~h}$ after injection of labeled lipoproteins, $21.6 \pm 1.8 \%$ of the initial amount of labeled Lp(a) and $42.5 \pm 2.3 \%$ of the initial amount of labeled LDL remained in plasma (Student's $t$ test: $P<0.001)$. The decay in plasma of ${ }^{125}$ I-labeled and ${ }^{131}$ I-labeled lipoproteins were very similar, both for $\mathrm{Lp}(\mathrm{a})$ and LDL, and there was no difference in decay of labeled Lp(a) or LDL between rabbits with nonlesioned aortas and rabbits with atherosclerotic aortas (data not shown). The decay in plasma of labeled Lp(a) was almost similar to that of unlabeled Lp(a) (Fig. 3).

Formation of labeled free apo(a) in plasma after injection of labeled $\mathrm{Lp}(\mathrm{a})$ was minimal: the fraction of radioactivity in labeled $\mathrm{Lp}(\mathrm{a})$ that migrated through anti-apo B-containing gel and precipitated in anti-Lp(a)-containing gel was $<1 \%, 10$ min, 3 and $26 \mathrm{~h}$ after injection of labeled Lp(a) (data not shown). In contrast, the fraction of total labeled $\mathrm{Lp}(\mathrm{a})$ in plasma that was present in $\mathrm{Lp}(\mathrm{a})$ - (i.e., labeled particles that migrated through anti-Lp(a)-containing gel and precipitated in anti-apo B-containing gel) increased with time during the $26 \mathrm{~h}$ of circulation in vivo (Fig. 3). In accordance with this and the fact that $\operatorname{Lp}(\mathrm{a})$ - is smaller than $\operatorname{Lp}(\mathrm{a}),{ }^{131} \mathrm{I}$-labeled lipoproteins in plasma $26 \mathrm{~h}$ after intravenous injection of ${ }^{131} \mathrm{I}-\mathrm{Lp}(\mathrm{a})$ eluted slightly later than ${ }^{125} \mathrm{I}$-labeled lipoproteins in plasma $3 \mathrm{~h}$ after injection of ${ }^{125} \mathrm{I}-\mathrm{Lp}$ (a) on gel filtration chromatography (Fig. 2).

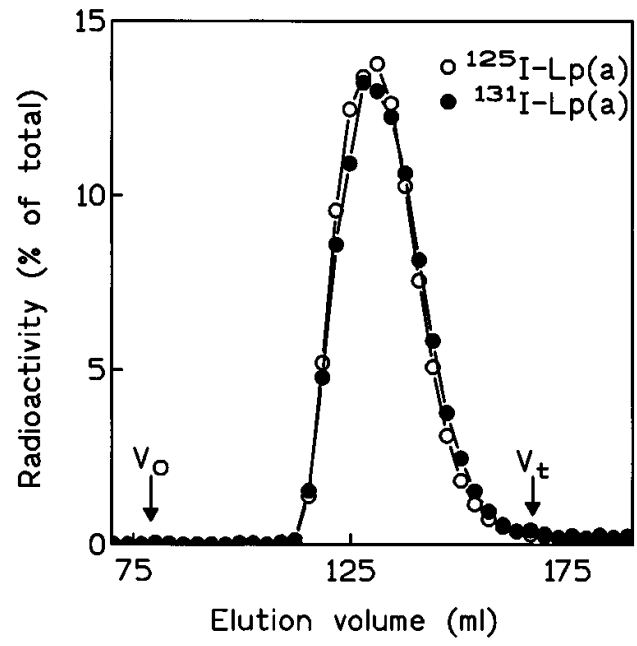

Figure 2. Gel filtration chromatography of rabbit plasma $3 \mathrm{~h}$ after intravenous injection of ${ }^{125} \mathrm{I}-\mathrm{Lp}(\mathrm{a})$ and $26 \mathrm{~h}$ after injection of ${ }^{131} \mathrm{I}-\mathrm{Lp}(\mathrm{a})$. The recovery was $99 \%$ for ${ }^{125} \mathrm{I}-\mathrm{Lp}(\mathrm{a})$ and $100 \%$ for ${ }^{131} \mathrm{I}-\mathrm{Lp}(\mathrm{a}) . \mathrm{V}_{0}$ and $\mathrm{V}_{\mathrm{t}}$ are void and total volume, respectively. Iodinated LDL eluted $\sim 5$ $\mathrm{ml}$ before $\mathrm{Lp}(\mathrm{a})$ on this column $(2.6 \mathrm{~cm} \times 100 \mathrm{~cm})$; the gel was Sephacryl S-500 HR (Pharmacia LKB Biotechnology Inc.).

Aortic cholesterol. The cholesterol content in the nonlesioned aortas from the rabbits that were fed cholesterol for 6-10 d (Fig. 4) was similar to the aortic cholesterol content in rabbits that had never been fed cholesterol (31). In the present study, the values were $2.2 \pm 0.2,1.9 \pm 0.3,2.1 \pm 0.3,1.8 \pm 0.3$, $1.6 \pm 0.3$, and $1.8 \pm 0.3 \mathrm{nmol} / \mathrm{mg}$ wet wt $(n=12)$ in segments 1 , $2,3,4,5$, and 6 , respectively. The cholesterol content of atherosclerotic aortas in rabbits that had been fed cholesterol for 173-236 d was markedly elevated compared with the cholesterol content of nonlesioned aortas. In atherosclerotic aortas, there was a highly significant variation in cholesterol content between aortic segments (ANOVA, effect of aortic site: $P<$ 0.0001). Most cholesterol accumulated in the aortic arch (Fig.

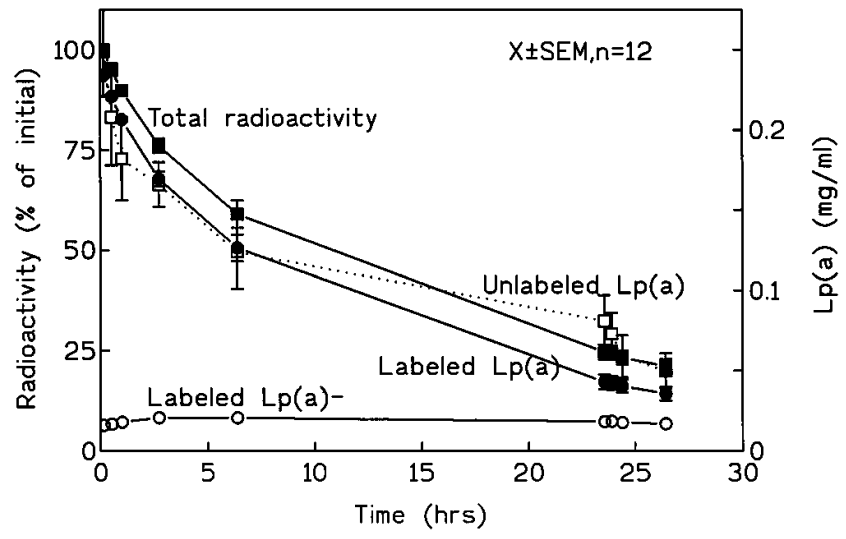

Figure 3. Plasma radioactivity decay curves (filled lines, left y-axis) and plasma $\mathrm{Lp}$ (a) mass (dotted line, right y-axis) after intravenous injection of ${ }^{131} \mathrm{I}-\mathrm{Lp}(\mathrm{a})$ and $d<1.12 \mathrm{~g} / \mathrm{m}$ lipoproteins. - $\mathbf{\square}$-, total radioactivity; - $\square-$, Lp(a) plasma concentration ( $\mathrm{mg} / \mathrm{ml})$; - - -, labeled Lp(a); -O-, labeled Lp(a) - . Values for labeled Lp(a) - were determined at $10 \mathrm{~min}, 3 \mathrm{~h}$, and $26 \mathrm{~h}$ and the relative amount of radioactivity and $\mathrm{Lp}(\mathrm{a})-$ at other time points was estimated by linear interpolation in each rabbit. Values are means \pm SEM. 


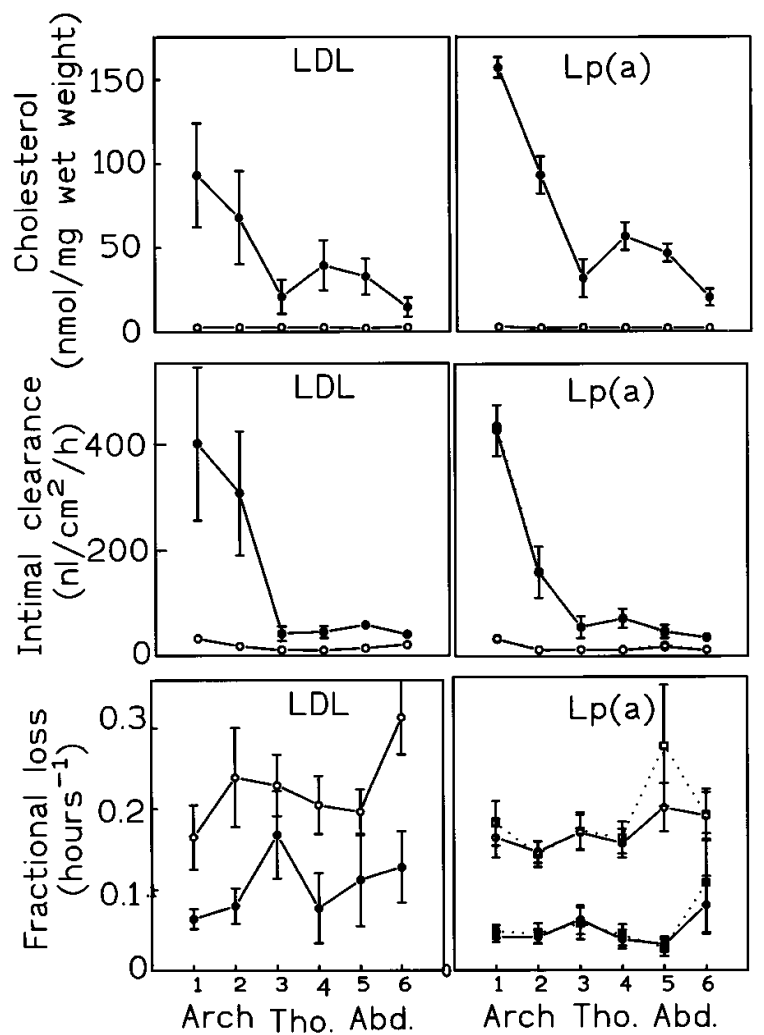

Aortic segments

Figure 4. Cholesterol content and lipoprotein intimal clearance and fractional loss in the intima-inner media of six aortic segments of nonlesioned $(\bigcirc)$ and atherosclerotic aortas $(\bullet)$ of the cholesterol-fed rabbits studied. Intimal clearance and fractional loss of labeled $\mathrm{Lp}(\mathrm{a})$ after corrections for $\mathrm{Lp}(\mathrm{a})$ - contribution to total values (circles) (see Methods) are shown as boxes connected by dotted lines. Values are means \pm SEM.

4). There was no significant difference in aortic cholesterol content in atherosclerotic aortas between rabbits used to study LDL and Lp(a) (ANOVA, effect of group of rabbits: $P=$ $0.10)$, but there was a difference in the variance between the two groups $(P<0.01)$.

Intimal clearance. Intimal clearance and fractional loss were calculated using a one-pool compartment model of the aortic intima-inner media. Intimal clearance represents the rate constant for transfer of a given species of labeled lipoproteins from plasma into the arterial wall.

The final ANOVA model to describe intimal clearance included aortic site $(P<0.0001)$, atherosclerosis $(P<0.0001)$, and the aortic site by atherosclerosis interaction $(P<0.0001)$. The intimal clearance of both lipoproteins was larger in atherosclerotic than in nonlesioned aortas (Fig. 4). The relatively larger intimal clearance in segments 1,2 , and 4 of atherosclerotic aorta compared with nonlesioned aorta was the cause of the aortic site by atherosclerosis interaction. There was no difference in intimal clearance between Lp(a) and LDL $(P<$ 0.90).

Fractional loss. Fractional loss represents the rate constant for loss of newly entered lipoprotein from the arterial intima-inner media, by degradation by arterial wall cells or by transfer of labeled lipoproteins out of the arterial wall.
The final ANOVA model to describe fractional loss included aortic site $(P<0.0001)$ and atherosclerosis $(P<$ 0.0001). On a logarithmic scale, the variance in fractional loss was larger in nonlesioned aortas than in atherosclerotic aortas; however, the model allows such a difference. Fractional loss in atherosclerotic intima-inner media of labeled $\mathrm{Lp}(\mathrm{a})$ and labeled LDL was on average 25 and $43 \%$, respectively, of that in nonlesioned intima-inner media (Fig. 4). Fractional loss of labeled lipoproteins was lower in the atherosclerosis-prone aortic arch than in the more atherosclerosis-resistant abdominal aorta.

Although type of lipoprotein did not remain in the final ANOVA model for fractional loss, fractional loss of $\mathrm{Lp}(\mathrm{a})$ was borderline significantly lower than that of LDL (ANOVA, effect of type of lipoprotein; $P=0.07$ ); $\mathrm{Lp}(\mathrm{a})$ fractional loss was $73 \%$ (95\% confidence, interval 51 to $104 \%$ ) of that of LDL. Since there was no interaction between type of lipoprotein and absence or presence of atherosclerosis, this borderline significant effect is derived both from nonlesioned and atherosclerotic aorta.

Crude accumulation. The final ANOVA model to describe crude accumulation during the $3 \mathrm{~h}$ included aortic site $(P<0.0001)$, atherosclerosis $(P<0.0001)$, and the aortic site by atherosclerosis interaction $(P<0.0001)$. On a logarithmic scale, the variance was larger in the aortic arch than in the thoracic and abdominal aortas $(P<0.0001)$, but the model was modified to allow this difference. The crude accumulation during the $3 \mathrm{~h}$ for both lipoproteins was larger in atherosclerotic than in nonlesioned aortas (Table II). The relatively larger accumulation in segments 1,2 , and 4 of the atherosclerotic aorta compared to the nonlesioned aorta was the cause of the aortic site by atherosclerosis interaction. There was no difference in accumulation during the $3 \mathrm{~h}$ between $\operatorname{Lp}(\mathrm{a})$ and $\operatorname{LDL}(P=$ 0.90).

The final ANOVA model to describe crude accumulation during the $26 \mathrm{~h}$ also included aortic site $(P<0.0001)$, atherosclerosis $(P<0.0001)$, and the aortic site by atherosclerosis interaction $(P<0.0001)$. On a logarithmic scale, the variance was larger for nonlesioned than for atherosclerotic aorta $(P<$ 0.0001). The crude accumulation during the $26 \mathrm{~h}$ for both lipoproteins was larger in atherosclerotic than in nonlesioned aortas (Table II). The relatively larger accumulation in segments 1,2 , and 4 of atherosclerotic aorta compared to nonlesioned aorta was the cause of the aortic site by atherosclerosis interaction. There was no significant difference in accumulation during the $26 \mathrm{~h}$ between Lp(a) and LDL $(P<0.10)$; the accumulation of $\mathrm{Lp}(\mathrm{a})$ was $121 \%$ (95\% confidence interval $95-152 \%)$ of that of LDL.

Crude fractional loss. The final ANOVA model to describe crude fractional loss included aortic site $(P<0.0005)$, atherosclerosis $(P<0.0001)$, and the aortic site by atherosclerosis interaction $(P<0.0001)$. On a logarithmic scale, the variance was larger for nonlesioned aorta than for atherosclerotic aorta $(P<0.0005)$. Crude fractional loss for both lipoproteins was smaller in atherosclerotic than in nonlesioned intima-inner media (Table II). There was no significant difference in crude fractional loss of $\operatorname{Lp}(\mathrm{a})$ and $\operatorname{LDL}(P<0.90)$.

\section{Discussion}

A noticeable finding of the present study was a markedly reduced fractional loss of $\mathrm{Lp}(\mathrm{a})$ and LDL in atherosclerotic com- 
Table II. Crude Accumulation After 3- and 26-h Exposures and Crude Fractional Loss of Labeled Lp(a) and Labeled LDL in Nonlesioned and Atherosclerotic Aorta

\begin{tabular}{|c|c|c|c|c|c|c|}
\hline & \multicolumn{6}{|c|}{ Aortic segment } \\
\hline & 1, Arch & 2, Arch & 3, Thoracic & 4, Thoracic & 5, Abdominal & 6, Abdominal \\
\hline \multicolumn{7}{|l|}{ Nonlesioned aorta } \\
\hline \multicolumn{7}{|c|}{ Crude accumulation during } \\
\hline $\mathrm{Lp}(\mathrm{a}), n=6$ & $1.59 \pm 0.25$ & $0.64 \pm 0.12$ & $0.71 \pm 0.06$ & $0.67 \pm 0.10$ & $0.99 \pm 0.19$ & $0.78 \pm 0.17$ \\
\hline LDL, $n=7$ & $1.99 \pm 0.30$ & $1.21 \pm 0.25$ & $0.81 \pm 0.17$ & $0.71 \pm 0.16$ & $1.18 \pm 0.37$ & $1.48 \pm 0.34$ \\
\hline \multicolumn{7}{|c|}{ Crude accumulation during } \\
\hline $\mathrm{Lp}(\mathrm{a}), n=6$ & $2.99 \pm 0.65$ & $1.29 \pm 0.29$ & $1.24 \pm 0.13$ & $1.18 \pm 0.15$ & $1.34 \pm 0.14$ & $1.07 \pm 0.13$ \\
\hline LDL, $n=7$ & $4.57 \pm 1.98$ & $1.98 \pm 0.40$ & $1.20 \pm 0.12$ & $1.12 \pm 0.10$ & $1.80 \pm 0.40$ & $1.68 \pm 0.32$ \\
\hline \multicolumn{7}{|c|}{$\begin{array}{c}\text { Crude fractional loss } \\
\text { (per } 26 \mathrm{~h})\end{array}$} \\
\hline $\mathrm{Lp}(\mathrm{a}), n=6$ & $0.77 \pm 0.04$ & $0.77 \pm 0.02$ & $0.79 \pm 0.03$ & $0.78 \pm 0.04$ & $0.85 \pm 0.04$ & $0.80 \pm 0.05$ \\
\hline LDL, $n=7$ & $0.71 \pm 0.06$ & $0.79 \pm 0.04$ & $0.80 \pm 0.04$ & $0.79 \pm 0.03$ & $0.78 \pm 0.04$ & $0.85 \pm 0.01$ \\
\hline \multicolumn{7}{|l|}{ Atherosclerotic aorta } \\
\hline \multicolumn{7}{|c|}{ Crude accumulation during } \\
\hline $\mathrm{Lp}(\mathrm{a}), n=6$ & $14.5 \pm 2.15$ & $6.28 \pm 1.27$ & $2.71 \pm 0.87$ & $3.35 \pm 0.70$ & $2.46 \pm 0.43$ & $2.01 \pm 0.34$ \\
\hline LDL, $n=6$ & $12.2 \pm 4.00$ & $10.8 \pm 4.06$ & $2.29 \pm 0.61$ & $2.36 \pm 0.44$ & $3.02 \pm 0.29$ & $2.51 \pm 0.34$ \\
\hline \multicolumn{7}{|c|}{ Crude accumulation during } \\
\hline $\mathrm{Lp}(\mathrm{a}), n=6$ & $71.8 \pm 8.90$ & $30.7 \pm 5.1$ & $12.6 \pm 4.21$ & $17.2 \pm 3.20$ & $14.7 \pm 2.79$ & $10.0 \pm 3.12$ \\
\hline LDL, $n=6$ & $52.8 \pm 19.4$ & $51.3 \pm 21.4$ & $13.4 \pm 7.32$ & $17.1 \pm 5.88$ & $15.6 \pm 6.22$ & $10.2 \pm 5.22$ \\
\hline \multicolumn{7}{|c|}{$\begin{array}{c}\text { Crude fractional loss } \\
\text { (per } 26 \mathrm{~h})\end{array}$} \\
\hline $\mathrm{Lp}(\mathrm{a}), n=6$ & $0.42 \pm 0.05$ & $0.39 \pm 0.09$ & $0.48 \pm 0.10$ & $0.37 \pm 0.09$ & $0.31 \pm 0.09$ & $0.45 \pm 0.15$ \\
\hline LDL, $n=6$ & $0.57 \pm 0.07$ & $0.51 \pm 0.05$ & $0.47 \pm 0.14$ & $0.24 \pm 0.14$ & $0.45 \pm 0.16$ & $0.55 \pm 0.17$ \\
\hline
\end{tabular}

Values are means \pm SEM.

pared with nonlesioned intima-inner media; the same conclusion was drawn when the much simpler calculation of crude fractional loss was done. This supports the idea that reduced fractional loss of both of these lipoproteins from arterial intima may play an important role during the development of atherosclerosis. The data also underscores the notion that an increased macromolecular intimal clearance, leading to an increased lipoprotein influx, contributes significantly to the accumulation of $\mathrm{Lp}(\mathrm{a})$ and LDL in atherosclerotic lesions.

In the present study, the difference in fractional loss of Lp(a) and LDL between nonlesioned and atherosclerotic aortas was similar at all aortic sites, while accumulation of cholesterol and severity of atherosclerosis in the intima was much more pronounced in the aortic arch than in the thoracic and abdominal aortas. It cannot therefore be excluded that the difference in fractional loss between normal and atherosclerotic aortas resulted from as yet uncharacterized general structural changes in the intima after long term cholesterol feeding rather than from the presence of atherosclerosis per se. The nature of such structural changes that would cause a decreased loss of lipoproteins from the intima-inner media is unknown. Increased diffusion distance due to intimal thickening may play a role. Previous in vivo studies on the effect of atherosclerosis on fractional loss of labeled LDL from the arterial wall are conflicting: one study in the pigeon found a significantly lower frac- tional loss of LDL from aortic sites with atherosclerosis than from adjacent nonatherosclerotic sites (32), a finding similar to the present study, whereas other studies in fat-fed monkeys (23) and genetically hyperlipidemic rabbits (22) did not detect differences between nonlesioned and atherosclerotic arteries. It cannot be excluded that differences in the composition of atherosclerotic plaques in aortas of cholesterol-fed rabbits and pigeons compared with that in aortas of fat-fed monkeys and genetically hyperlipidemic rabbits have induced the discrepant findings. It is, however, possible that the two studies with negative results $(22,23)$ overlooked a difference between normal and atherosclerotic aortas because of limited statistical power.

The importance of plasma contamination on the arterial surface for interpretation of the present aortic radioactivity data was estimated by considering a worst-case situation, in which as much as $50 \%$ of the radioactivity in the intima-inner media of the nonlesioned aortas after $3 \mathrm{~h}$ exposure to labeled Lp(a) or labeled LDL was attributable to plasma contamination (in atherosclerotic aorta, plasma contamination corrections would only have a minimal effect because the intimal clearance was very large). This estimate yielded plasma contaminations ranging from as much as an average $43 \mathrm{nl} / \mathrm{cm}^{2}$ in the nonlesioned proximal segment of the aortic arch to an average $15 \mathrm{nl} / \mathrm{cm}^{2}$ in the distal segment of the thoracic aorta. These plasma contaminations of aortic intima-inner media are 
considerably higher than the average $6 \mathrm{nl} / \mathrm{cm}^{2}$ in a previous study (31), but similar to an average $30 \mathrm{nl} / \mathrm{cm}^{2}$ in another study (15), both from our laboratory. After subtraction of aortic radioactivity after 3- and 26-h exposures estimated to be due to plasma contamination in each aortic segment of each rabbit, fractional loss of labeled $\mathrm{Lp}(\mathrm{a})$ and LDL from nonlesioned aortas were recalculated: fractional loss in atherosclerotic aortic intima-inner media of labeled Lp(a) and labeled LDL was then on average 51 and $78 \%$, respectively, of that in nonlesioned aortic intima-inner media. Accordingly, the overall conclusion of a reduced fractional loss of Lp(a) and LDL in atherosclerotic compared to nonlesioned aortas, is valid even after these plasma contamination corrections.

In the present study, fractional loss of labeled Lp(a) and labeled LDL from nonlesioned aortas was lowest in the atherosclerosis-susceptible aortic arch and highest in the atherosclerosis-resistant abdominal aorta. This is in accordance with a previous study by Schwenke and Carew (33). These findings support the idea that regional variations in rates of loss of LDL and $\mathrm{Lp}(\mathrm{a})$ in the intima may be important for the focal development of atherosclerotic lesions, at least in the initial stages of the atherogenesis (33). The extracellular matrix most likely plays a role in lipoprotein retention by aortic tissue (34), and LDL has been shown to bind more to types I and III collagen than to types IV and V collagen (35). Moreover, glycosaminoglycan fractions from human arteries presenting diverse susceptibilities to atherosclerosis have different binding affinities to LDL (36). Thus, variation in fractional loss of lipoproteins could be related to variation in affinity of aortic glycosaminoglycans to LDL and Lp(a) at different aortic sites.

Previous studies have suggested that $\mathrm{Lp}(\mathrm{a})$ accumulates in vessel walls preferentially to LDL (6-8) and in vitro studies suggest that $\mathrm{Lp}(\mathrm{a})$ relative to LDL has a higher affinity to fibrin (37) and to arterial wall glycosaminoglycans (38). Moreover, we have recently reported a markedly increased accumulation of Lp(a) compared with LDL in balloon-injured rabbit aorta in vivo (13). In the present study, there was a borderline significant lower fractional loss for labeled $\mathrm{Lp}(\mathrm{a})$ than for labeled LDL in nonlesioned and atherosclerotic aortas combined. However, since atherosclerotic aortas used for studying $\mathrm{Lp}$ (a) had (nonsignificantly) more cholesterol than aortas used for studying LDL (Fig. 4), and since atherosclerosis reduces fractional loss, it is possible that the difference in fractional loss between Lp(a) and LDL can be explained by differences in the severity of atherosclerosis.

One explanation for the present lack of a clear difference in fractional loss between Lp(a) and LDL may be that the rabbit aorta does not contain sufficient amounts of structural components, such as fibrin and glycosaminoglycans, which may be necessary for specific accumulation of $\mathrm{Lp}$ (a) compared with LDL in the intima. A second explanation could be that an experimental period of $26 \mathrm{~h}$ is too short to detect subtle differences in loss of $\mathrm{Lp}(\mathrm{a})$ and LDL from the arterial wall. Finally, the one-pool compartment model may not adequately describe the kinetics of $\mathrm{Lp}(\mathrm{a})$ in the arterial wall. Nevertheless, calculation of crude fractional loss that does not assume a one-pool compartment model also failed to demonstrate a statistically significant difference in losses of Lp(a) and LDL.

The intimal clearance was similar for $\mathrm{Lp}(\mathrm{a})$ and $\mathrm{LDL}$ throughout the length of aorta. This observation suggests that the transfer of $\mathrm{Lp}(\mathrm{a})$ from plasma across the endothelial barrier is by the same mechanism as LDL; i.e., presumably a lipo- protein size, lipoprotein concentration, and blood pressuredependent transfer $(15,39,40)$, independent of LDL-receptors (41). The present data are in accordance with findings from our previous study in which there was a close association between the accumulation of labeled Lp(a) and LDL during 1 and $3 \mathrm{~h}$ in rabbit aorta after labeled Lp(a) and LDL were injected simultaneously (21).

In accordance with our previous studies in rabbits, the plasma decay of $\operatorname{Lp}(\mathrm{a})$ was faster than that of LDL (21), which contrasts observations in humans (42-44). Importantly, using iodinated Lp(a) and LDL that had been labeled using the same protocol as the present study, we found a similar removal rate of simultaneously injected $\mathrm{Lp}(\mathrm{a})$ and LDL in humans during a 3-h observation period (data will be published elsewhere). Furthermore, in the present study, plasma decay of labeled and unlabeled Lp(a) after a bolus injection were almost similar. These data altogether support the conclusion that iodination did not severely affect the metabolism of $\mathrm{Lp}(\mathrm{a})$.

Rabbits injected with labeled $\mathrm{Lp}$ (a) additionally received a bolus injection of unlabeled $\mathrm{Lp}(\mathrm{a})$ at the start of the experiment to increase the plasma levels of $\mathrm{Lp}(\mathrm{a})$ to a level comparable to that in humans. The decline in plasma Lp(a) concentration during the experiment is not believed to have affected the present results, since the accumulation of labeled $\mathrm{Lp}(\mathrm{a})$ in the rabbit aortic intima-inner media was similar after a 5-10-min (13), 3-h (21), and 23-h exposure (12), irrespective of whether the rabbits had received a bolus injection of unlabeled $\mathrm{Lp}(\mathrm{a})$ together with labeled $\mathrm{Lp}(\mathrm{a})$ or not.

The fraction of total plasma radioactivity in $\mathrm{Lp}(\mathrm{a})-$ increased with time after intravenous injection of labeled $\mathrm{Lp}(\mathrm{a})$. The contribution of $\mathrm{Lp}(\mathrm{a})$ - to the total area under the plasma radioactivity concentration vs the time curve during the $26 \mathrm{~h}$ after injection of labeled $\mathrm{Lp}(\mathrm{a})$ was on average $16 \%$. The contribution of this labeled $\mathrm{Lp}(\mathrm{a})$ - to the total arterial wall radioactivity was assessed under the assumption that $\mathrm{Lp}(\mathrm{a})-$ interacts with the arterial wall like LDL (see Methods). Corrections for $\mathrm{Lp}(\mathrm{a})$ - changed the values of intimal clearance and fractional loss of labeled $\mathrm{Lp}(\mathrm{a})$ only to a minimal extent and did not affect the overall results and conclusions of the present study. No labeled free apo(a) was detected in plasma, either on two-tier rocket immunoelectrophoresis or on gel filtration chromatography. This observation is similar to that in humans after an intravenous injection of iodinated $\operatorname{Lp}(\mathrm{a})(28,42)$, and is particularly relevant for the present study since free apo(a), due to its small size similar to albumin and HDL, presumably would enter and leave the arterial wall more rapidly than $\operatorname{Lp}(\mathrm{a})(22,39)$. We have previously estimated that 15 to $25 \%$ of the radioactivity in iodinated $\mathrm{Lp}(\mathrm{a})$ is in apo(a), with the remaining fraction in apo B (21). Formation of labeled Lp(a)must therefore be accompanied by the formation of labeled free apo(a). Since labeled free apo(a), however, was not detectable in plasma, labeled free apo(a) must either be removed very rapidly from plasma or be dissociated from Lp(a) extravascularly. If labeled free apo(a) was dissociated from $\mathrm{Lp}(\mathrm{a})$ in the arterial wall and labeled free apo(a) was then removed from the wall faster than $\operatorname{Lp}(\mathrm{a})-$, this may have led to an overestimation of fractional loss of the lipid/apo B moiety of $\operatorname{Lp}(\mathrm{a})$.

In conclusion, the present data suggest that decreased fractional loss of LDL and Lp(a) in the intima at atherosclerosissusceptible sites accompanies the development of atherosclerosis. The data further suggest that $\mathrm{Lp}(\mathrm{a})$ shares with LDL the 
potential for causing atherosclerosis; the increased intimal clearance and decreased fractional loss of $\mathrm{Lp}(\mathrm{a})$ in atherosclerotic aortas favors the idea of accumulation of $\mathrm{Lp}(\mathrm{a})$ in atherosclerotic lesions. This may stimulate foam cell formation (45), smooth muscle cell proliferation (46), and fibrin deposition (47).

\section{Acknowledgments}

The authors thank Thomas Scheike, PhD, Dept. of Biostatistics, University of Copenhagen for thorough help in the statistical analysis. Karen Rasmussen and Kurt S. Jensen provided excellent technical assistance.

This study was supported by the Danish Medical Research Council, and the Danish foundations Novo's Fond Komite and Overlæge Johan Boserup og Lise Boserups Legat.

\section{References}

1. Pyörälä, K., G. De Backer, I. Graham, P. Poole-Wilson, and D. Wood. 1994. Prevention of coronary heart disease in clinical practice. Recommendations of The Task Force of the European Society of Cardiology, European Atherosclerosis Society and European Society of Hypertension. Eur. Heart J. 15: $1300-1331$.

2. Rader, D.J., J.M.. Hoeg, and H.B. Brewer, Jr. 1994. Quantitation of plasma apolipoproteins in the primary and secondary prevention of coronary artery disease. Ann. Intern. Med. 120:1012-1025.

3. Lawn, R.M., D.P. Wade, R.E. Hammer, G. Chiesa, J.G. Verstuyft, and E.M. Rubin. 1992. Atherogenesis in transgenic mice expressing human apolipoprotein(a). Nature (Lond.). 360:670-672.

4. Rath, M., A. Niendorf, T. Reblin, M. Dietel, H. Krebber, and U. Beisiegel. 1989. Detection and quantification of lipoprotein(a) in the arterial wall of 107 coronary bypass patients. Arteriosclerosis. 9:579-592.

5. Niendorf, A., M. Rath, K. Wolf, S. Peters, H. Arps, U. Beisiegel, and M. Dietel. 1990. Morphological detection and quantification of lipoprotein(a) deposition in atheromatous lesions of human aorta and coronary arteries. Virchows Archiv. Abt. A. Pathol. Anat. 417:105-111.

6. Cushing, G.L., J.W. Gaubatz, M.L. Nava, B.J. Burdick, T.M.A. Bocan, J.R. Guyton, D. Weilbaecher, M.E. DeBakey, G.M. Lawrie, and J.D. Morrisett. 1989. Quantitation and localization of apolipoprotein [a] and B in coronary artery bypass vein grafts resected at reoperation. Arteriosclerosis. 9:593-603.

7. Smith, E.B. and S. Cochran. 1990. Factors influencing the accumulation in fibrous plaques of lipid derived from low density lipoprotein. II. Preferential immobilization of lipoprotein(a) (Lp(a)). Atherosclerosis. 84:173-181.

8. Kreuzer, J., M.B. Lloyd, D. Bok, G.M. Fless, A.M. Scanu, A.J. Lusis, and M.E. Haberland. 1994. Lipoprotein(a) displays increased accumulation compared with low-density lipoprotein in the murine arterial wall. Chem. Phys. Lipids. 67/68:175-190.

9. Stary, H.C., A.B. Chandler, S. Glagov, J.R. Guyton, W. Insull, Jr., M.E. Rosenfeld, S.A. Schaffer, C.J. Schwartz, W.D. Wagner, and R.W. Wissler. 1994. A definition of initial, fatty streak, and intermediate lesions of atherosclerosis. A report from The Committee on Vascular Lesions of the Council of Arteriosclerosis, American Heart Association. Arterioscler. Thromb. 14:840-856.

10. Jacobsson, L.S., K. Persson, G. Aberg, R.G.G. Andersson, B.E. Karlberg, and A.G. Olsson. 1994. Antiatherosclerotic effects of the angiotensin-converting enzyme inhibitors captopril and fosinopril in hypercholesterolemic minipigs. J. Cardiovasc. Pharmacol. 24:670-677.

11. Hansen, B.F., A. Mortensen, J.F. Hansen, P. Ibsen, H. Frandsen, and B.G. Nordestgaard. 1994. Atherosclerosis in Watanabe heritable hyperlipidemic rabbits. Evaluation of macroscopic, microscopic and biochemical methods and comparison of atherosclerosis variables. APMIS. 102:177-190.

12. Zilversmit, D.B., and T.M. Shea. 1989. Quantitation of apoB-48 and apoB-100 by gel scanning or radio-iodination. J. Lipid Res. 30:1639-1646.

13. Nielsen, L.B., S. Stender, K. Kjeldsen, and B.G. Nordestgaard. 1996. Specific accumulation of Lipoprotein(a) in balloon-injured rabbit aorta in vivo. Circ. Res. 78:615-626.

14. Utermann, G., H.J. Menzel, H.G. Kraft, H.G. Kemmler, and C. Seitz. 1987. $\mathrm{Lp}$ (a) glycoprotein phenotypes. Inheritance and relation to $\mathrm{Lp}(\mathrm{a})-$ lipoprotein concentrations in plasma. J. Clin. Invest. 80:458-465.

15. Nielsen, L.B., S. Stender, K. Kjeldsen, and B.G. Nordestgaard. 1994. Effect of angiotensin II and enalapril on transfer of low-density lipoprotein into aortic intima in rabbits. Circ. Res. 75:63-69.

16. Chapman, M.J. 1986. Comparative analysis of mammalian plasma lipoproteins. Methods Enzymol. 128:70-143. Table V.

17. McFarlane, A.S. 1958. Efficient trace-labelling of proteins with iodine. Nature (Lond.). 182:53.

18. Bilheimer, D.W., S. Eisenberg, and R.I. Levy. 1972. The metabolism of very low density lipoprotein proteins I. Preliminary in vitro and in vivo observa- tions. Biochim. Biophys. Acta. 260:212-221.

19. Folch, J., M. Lees, and G.H. Sloane Stanley. 1957. A simple method for the isolation and purification of total lipids from animal tissues. J. Biol. Chem. 226:497-509.

20. Ness, A.T., J.V. Pastewka, and A.C. Peakock. 1964. Evaluation of a recently reported stable Liebermann-Burchard reagent and its use for the direct determination of serum total cholesterol. Clin. Chim. Acta. 10:229-237.

21. Nielsen, L.B., B.G. Nordestgaard, S. Stender, A. Niendorf, and K Kjeldsen. 1995. Transfer of lipoprotein(a) and LDL into aortic intima in normal and in cholesterol-fed rabbits. Arterioscler. Thromb. Vasc. Biol. 15:1492-1502.

22. Nordestgaard, B.G., R. Wootton, and B. Lewis. 1995. Selective retention of VLDL, IDL, and LDL in the arterial intima of genetically hyperlipidemic rabbits in vivo. Molecular size as a determinant of fractional loss from intima-inner media. Arterioscler. Thromb. Vasc. Biol. 15:534-542.

23. Ghosh, S., M.L. Armstrong, M.B. Megan, and F.H. Cheng. 1987. Arterial uptake indices of low density lipoproteins after fatty streak formation in Cynomolgus monkeys. Cardiovasc. Res. 21:14-20.

24. Wootton, R., P. Baskerville, P. Turner, M. Insell, M. Shaik, A. La Ville, J. Quiney, N.L. Browse, and B. Lewis. 1987. A method for quantifying lipoprotein flux rates between plasma and arterial intima in vivo. Clin. Phys. Physiol Meas. 8:65-74.

25. Schwenke, D.C., and D.B. Zilversmit. 1989. The arterial barrier to lipoprotein influx in the hypercholesterolemic rabbit. 1. Studies during the first two days after mild aortic injury. Atherosclerosis. 77:91-103.

26. Schwenke, D.C., and D.B. Zilversmit. 1987. Enhanced accumulation and turnover of esterified cholesterol in injured rabbit aorta. Arteriosclerosis. 7 : 367-377.

27. Fless, G.M., M.E. ZumMallen, and A.M. Scanu. 1985. Isolation of apolipoprotein(a) from lipoprotein(a). J. Lipid. Res. 26:1224-1229.

28. Rader, D.J., W.A. Mann, W. Cain, H.G. Kraft, D. Usher, L.A. Zech, J.M. Hoeg, J. Davignon, P. Lupien, M. Grossmann, et al. 1995. The low density lipoprotein receptor is not required for normal catabolism of $\mathrm{Lp}(\mathrm{a})$ in humans. J. Clin. Invest. 95:1403-1408.

29. Bickel, P.J., and K.A. Doksum. 1977. Mathematical Statistics. Basic Ideas and Selected Topics. Holden-Day Series in Probability and Statistics. Holden-Day Inc., Oakland, CA. 283 pp.

30. Jennich, R.I., and M.D. Schluchter. 1986. Unbalanced repeated-measures models with structured covariance matrices. Biometrics. 42:805-820.

31. Nielsen, L.B., B.G. Nordestgaard, S. Stender, and K. Kjeldsen. 1992. Aortic permeability to LDL as a predictor of aortic cholesterol accumulation in cholesterol-fed rabbits. Arterioscler. Thromb. 12:1402-1409.

32. Schwenke, D.C., and R.W. St. Clair. 1993. Influx, efflux, and accumulation of LDL in normal arterial areas and atherosclerotic lesions of White Carneau pigeons with naturally occurring and cholesterol aggravated aortic atherosclerosis. Arterioscler. Thromb. 13:1368-1381.

33. Schwenke, D.C., and T.E. Carew. 1989. Initiation of atherosclerotic lesions in cholesterol-fed rabbits. II. Selective retention of LDL vs. selective in creases in LDL permeability in susceptible sites of arteries. Arteriosclerosis. 9: 908-918.

34. Srinivasan, S.R., P. Vijayagopal, E.R. Dalferes, Jr, B. Abbate, B Radhakrishnamurthy, and G.S. Berenson. 1986. Low density lipoprotein retention by aortic tissue. Contribution of extracellular matrix. Atherosclerosis. 62: 201-208.

35. Jimi, S., N. Sakata, A. Matunaga, and S. Takebayashi. 1994. Low density lipoproteins bind more to type I and III collagens by negative charge-dependent mechanisms than to type IV and V collagens. Atherosclerosis. 107:109-116.

36. Cardoso, L.E.M., and P.A.S. Mourao. 1994. Glucosaminoglycan fractions from human arteries presenting diverse susceptibilities to atherosclerosis have different binding affinities to plasma LDL. Arterioscler. Thromb. 14:115124.

37. Fless, G.M., and M.L. Snyder. 1994. Polymorphic forms of Lp(a) with different structural and functional properties: cold-induced self-association and binding to fibrin and lysine-sepharose. Chem. Phys. Lipids. 67/68:69-79.

38. Bihari-Varga, M., E. Gruber, M. Rotheneder, R. Zechner, and G.M. Kostner. 1988. Interaction of lipoprotein lp(a) and low density lipoprotein with glycosaminoglycans from human aorta. Arteriosclerosis. 8:851-857.

39. Stender, S., and D.B. Zilversmit. 1981. Transfer of plasma lipoprotein components and of plasma proteins into aortas of cholesterol-fed rabbits: molecular size as a determinant of plasma lipoprotein influx. Arteriosclerosis. 1:3849.

40. Nordestgaard, B.G., A. Tybjærg-Hansen, and B. Lewis. 1992. Influx in vivo of low density, intermediate density, and very low density lipoproteins into aortic intimas of genetically hyperlipidemic rabbits. Roles of plasma concentration, extent of aortic lesion, and lipoprotein particle size as determinants. Arterioscler. Thromb. 12:6-18.

41. Wiklund, O., T.E. Carew, and D. Steinberg. 1985. Role of the low density lipoprotein receptor in penetration of low density lipoprotein into rabbit aortic wall. Arteriosclerosis. 5:135-141.

42. Knight, B.L., Y.F.N. Perombelon, A.K. Soutar, D.P. Wade, and M. Seed. 1991. Catabolism of lipoprotein(a) in familial hypercholesterolemic subjects. Atherosclerosis. 87:227-237.

43. Krempler, F., G.M. Kostner, A. Roscher, F. Haslauer, and K. Bolzano. 
1983. Studies on the role of specific cell surface receptors in the removal of lipoprotein (a) in man. J. Clin. Invest. 71:1431-1441.

44. Rader, D.J., W. Cain, K. Ikewaki, G. Talley, L.A. Zech, D. Usher, and H.B. Brewer, Jr. 1994. The inverse association of plasma lipoprotein(a) concentrations with apolipoprotein(a) isoform size is not due to differences in $\mathrm{Lp}(\mathrm{a})$ catabolism but to differences in production rate. J. Clin. Invest. 93:2758-2763.

45. Bottalico, L.A., G.A. Keesler, G.M. Fless, and I. Tabas. 1993. Cholesterol loading of macrophages leads to marked enhancement of native lipopro- tein(a) and apoprotein(a) internalization and degradation. J. Biol. Chem. 268: 8569-8573.

46. Grainger, D.J., H.L. Kirschenlohr, J.C. Metcalfe, P.L. Weissberg, D.P Wade, and R.M. Lawn. 1993. Proliferation of human smooth muscle cells promoted by lipoprotein(a). Science (Wash. DC). 260:1655-1658.

47. Pizzo, S.V., and G.C. Howard. 1993. Lipoprotein(a) and its role in atherothrombotic disease. Lab. Invest. 69:373-386. 